\title{
Dissociation of Tetrameric Ions of Noncovalent Streptavidin Complexes Formed by Electrospray Ionization
}

\author{
Brenda L. Schwartz, James E. Bruce, Gordon A. Anderson, Steven A. \\ Hofstadler, Alan L. Rockwood, and Richard D. Smith \\ Chemical Methods and Separations Group, Chemical Sciences Department, Pacific Northwest Laboratory \\ Richland, Washington, USA
}

Ashutosh Chilkoti and Patrick S. Stayton

Center for Bioengineering, University of Washington Seattle, Washington

The noncovalent tetrameric association of the protein streptavidin formed by electrospray ionization (ESI) mass spectrometry has been observed intact and dissociated in the gas phase. An extended mass-to-charge ratio range quadrupole mass spectrometer was employed to examine the effects of harsher conditions in the ESI atmosphere-vacuum interface region on the streptavidin tetramer. Thermally induced dissociation caused the mass spectra to exhibit a series of complementary monomer and trimer ions that correspond to decomposition of the tetrameric species. Similar results were obtained with tandem mass spectrometric experiments on a Fourier transform ion cyclotron resonance mass spectrometer by application of sustained off-resonance irradiation (SORI) on a selected tetrameric charge state. The technique of single-frequency quadrupole excitation was used to accomplish selected-ion accumulation of the $14+$ charge state of the tetramer during ion injection. Subsequent low energy SORI combined with broadband quadrupole cooling produced the $7+$ monomer and $7+$ trimer species, as well as the $6+$ monomer and $8+$ trimer complementary ions. The observed asymmetric breakup of the tetramer is qualitatively explained by using physical models. (J Am Soc Mass Spectrom 1995, 6, 459-465)

$\mathrm{d}_{\mathrm{i}}$ lectrospray ionization mass spectrometry (ESIMS) has the potential to be a powerful technique in the analysis of liquid-phase noncovalent complexes [1]. Biomolecules that form structurally specific associations in solution have been shown to be capable of being preserved intact by the electrospray process for subsequent detection and investigation by mass spectrometry. Even relatively weak associations in solution can be observed by ESI-MS given the proper solution conditions and adjustment of the instrumental variables in the electrospray atmosphere-vacuum interface region. In the case of large proteins composed of multisubunits, the observation of intact quaternary associations by ESI-MS has proven to be a compromise between providing adequate heating-activation for desolvation of the large protein ions and maintaining mild enough interface conditions for mass spectrometric detection of the intact complex $[2,3]$. Noncovalent multimeric proteins electrosprayed from near-neutral $\mathrm{pH}$ aqueous solutions has been shown to be amenable

Address reprint requests to Richard D. Smith, Chemical Methods and Separations Group, Chemical Sciences Department, Pacific Northwest Laboratory, P. O. Box 999, Mail Stop P8-19, Richland, WA 99352. to the preservation of higher order structural associations, with the consequence of more limited charging for the molecular ion in the gas phase than observed under more conventional electrospray ionization (ESI) conditions [2, 3]. Therefore, more compact solution structures and imposed coulombic constraints can result in the formation of multimeric protein ions at high mass-to-charge ratio with relatively narrow charge state distributions [3]. It has been shown, at least in some cases, that the observation of ions that correspond to the intact multimeric associations requires the use of mass spectrometers capable of extended mass-tocharge ratio range $(>3000)$ detection.

Induction of decompositions of ions is a commonly accepted mass spectrometric approach to obtain information with regard to their structure in the gas phase. In ESI-MS, there are several ways to promote ion desolvation-dissociation, most generally by either thermal or collisional excitation. The simplest approach involves manipulation of variables in the atmosphere-vacuum interface region [1i, 4]. For example, increased heat applied to an inlet capillary readily allows thermally induced dissociation (TID) of noncovalent species [4c]. Collision-induced dissocia- 
tion (CID) also can be accomplished in the ESI interface region by increased voltage offset between the capillary and skimmer lens ( $\triangle \mathrm{CS}$ ) [4a]. Furthermore, tandem mass spectrometry experiments can be performed by selection of a specific charge state to undergo decomposition for elucidation of dissociation pathways. Information gathered from these studies could lend further insight into the electrospray process and the relationship of the gas-phase structure of ions to that in solution.

The protein streptavidin $\left(M_{\mathrm{r}} \sim 60 \mathrm{ku}\right)$ isolated from the bacterium Streptomyces avidinii exists in its active form as a noncovalent tetramer [5]. The four identical subunits of the native protein are each composed of a 159-residue polypeptide chain, contain a ligand binding site, and are devoid of carbohydrate. Streptavidin, along with the protein avidin, has an extremely selective and strong affinity for the ligand biotin $\left(K_{d} \sim\right.$ $10^{-15} \mathrm{M}$ ), which has led to the prevalent use of these noncovalent complex systems in bioanalytical applications [6]. Recently the intact tetrameric forms of the noncovalent avidin-biotin and streptavidin-biotin complexes were observed by ESI-MS [7]. Observation of these large protein noncovalent associations by ESIMS required a mass spectrometer capable of high mass-to-charge ratio detection because the relatively low charge states of the (apparently) compact structures for the tetrameric ions appeared in the $m / z$ $3500-4500$ range.

The active noncovalent tetrameric associations of several large proteins, which include concanavalin $A$ (Con $\mathrm{A})$, adult human hemoglobin $\left(\mathrm{Hb} \mathrm{A}_{\mathrm{o}}\right.$ ), and avidin, also previously have been observed intact by using our extended mass-to-charge ratio range single quadrupole mass spectrometer [3]. Given the proper solution conditions and gentle ESI interface conditions, the intact specific complexes were detected, consistent with predicted solution behavior, in preference to other nonspecific forms of aggregation that might have formed under some interface conditions during the ESI process. Either thermal or collisional activation in the interface region was found to readily dissociate these tetrameric species, which confirmed their noncovalent nature. Earlier studies conducted on the Con A tetramer [2] revealed that under certain interface conditions, the quaternary structure of the protein was lost, apparently prior to complete desolvation, and only higher charge state monomer ions were observed. This behavior suggested that protein charging in the gas phase was not conserved as the interface conditions were varied. Further studies [3] performed on the tetrameric forms of Con $\mathrm{A}, \mathrm{Hb} \mathrm{A}_{\mathrm{o}}$, and avidin illustrated that the implementation of harsher interface conditions produced a series of higher charge state monomer ions and surprisingly a series of low charge state (high mass-to-charge ratio) ions that could be attributed only to a trimer species (though trimers are not known to be formed under physiological conditions). Once formed, these trimers proved to be very stable to additional activation in the interface region. Electrospray mass spectra of solutions of Con A and $\mathrm{Hb} \mathrm{A}_{\mathrm{o}}$ where the dimeric form predominated yielded only monomer ions under harsher interface conditions. These observations led to the hypothesis that the trimers, because of their relatively decreased extent of charging, appearance at high mass-to-charge ratio, and stable nature once formed, resulted from decomposition of the tetramer late in the ion formationdesolvation process, that is, in the gas phase. Unfortunately, tandem mass spectrometry experiments were not possible with the extended mass-to-charge ratio instrument to prove that the trimer was a product of the gas-phase decomposition of the tetramer.

Fourier transform ion cyclotron resonance (FTICR) mass spectrometry coupled with ESI has proven to be a powerful tool in the analysis of biomolecules [8]. Providing the advantages of ultrahigh resolution, high sensitivity, and MS" (where $n \geq 2$ ) capabilities, FTICR permits the investigation of gas-phase structure of electrosprayed protein ions in addition to accurate molecular weight determination. We report observation of the intact noncovalent tetrameric association of the protein streptavidin by FTICR. In addition, we investigate dissociations of the tetrameric ions by CID on a FTICR instrument and correlate the results with TID experiments performed on the extended mass-tocharge ratio range single quadrupole mass spectrometer. CID studies were performed on a tetrameric charge state for streptavidin by using selected-ion accumulation [9] with subsequent sustained off-resonance irradiation (SORI) [10] in conjunction with broadband quadrupole excitation (axialization) [11]. The advantages of the combination of single-frequency quadrupole excitation and simultaneous external injection of electrosprayed ions for mass-to-charge ratio selected-ion accumulation have been reported previously [9]. With this technique, significant enhancement of ion signals, as well as dynamic range, is achieved by combination of the ion injection and quadrupole excitation events to facilitate the collection of ions of only one charge state in the cell. The implementation of SORI has been demonstrated to be an effective means for the study of the dissociation of ions by FTICR compared to conventional CID methods [10]. Application of an "off-resonance" activation frequency $500-2000 \mathrm{~Hz}$ away from the observed ion cyclotron frequency in the presence of gas allows large ions to experience many collisions over longer periods while they remain in the cell. Increased rf amplitude or decreased frequency difference of the cyclotron frequency and the SORI pulse allows more energy to be deposited into an ion to increase the amount of fragmentation. Recently, the benefits produced by implementation of broadband quadrupole excitation simultaneously with SORI, such as enhanced product ion detection efficiency and resolving power, were demonstrated [12]. However, for the present work, instead of a resistive network, the circuitry used to produce the 
two excitation events encompasses three wideband transformers [13]. These studies support previous results and provide additional insights into the dissociation of tetrameric proteins.

\section{Experimental}

The isolation and affinity purification of core recombinant streptavidin that incorporates residues $13-139$ is described in detail elsewhere [14]. Protein concentrations were $0.8 \mu \mathrm{g} \mu \mathrm{L}^{-1}$ in $10-\mathrm{mM}$ ammonium acetate ( $\mathrm{pH}$ 8.6).

Experiments performed to study ESI interface effects were conducted on a low frequency extended mass range single quadrupole mass spectrometer (Extrel, Pittsburgh, PA), developed in our laboratory; it has an approximate limit of $\mathrm{m} / \mathrm{z} 45,000$ [15]. The typical resolution for this low rf mass spectrometer is less than 100. A Teknivent Vector One data system was used for data acquisition. Spectra were acquired over a $\mathrm{m} / \mathrm{z} 500-10,000$ range at $\mathrm{m} / \mathrm{z} 5$ step sizes, which corresponded to approximately 2 min per scan, with the accumulation of five averaged scans. Mass axis calibration was performed with horse heart cytochrome $c$ in 10-mM ammonium acetate ( $\mathrm{pH}$ 6.7) by using the $12+$ to $1+$ charge states. The ESI interface consisted of a resistively heated stainless steel capillary (1.59-mm o.d., $0.5-\mathrm{mm}$ i.d., $20 \mathrm{~cm}$ long) similar to the design of Chait and co-workers [16] and others [4c]. Important interface conditions include the capillary heating applied across the capillary (reported as watts), the approximate temperature as measured on the outside surface of the capillary, and the voltage offset between the capillary and skimmer lens ( $\Delta C S$ ). A heated countercurrent flow of $\mathrm{N}_{2}$ gas was employed to assist electrospray desolvation. The electrospray source implemented in these studies did not utilize a sheath flow and allowed low flow rates $\left(0.3 \mu \mathrm{L} \min ^{-1}\right)$ [17].

The FTICR mass spectrometer consists of a 7-T superconducting magnet and is equipped with a modified external Analytica of Branford (Branford, CT) ESI source that contains a heated metal capillary [18]. Electrosprayed ions were injected into the ion cyclotron resonance (ICR) cell through two sets of rf-only quadrupoles and trapped with the assistance of a nitrogen gas pulse. The high pumping speed $\left(\sim 10^{5}\right.$ $\mathrm{L} / \mathrm{s}$ ) in the cell vacuum chamber was provided by cryopanels [18] that allowed rapid cycling between the high pressures $\left(10^{-4}\right.$ torr) needed for efficient ion accumulation and low pressures $\left(<10^{-9}\right.$ torr) for high performance detection. Cell trapping voltages were set high $(8-10 \mathrm{~V})$ to match the average kinetic energy of the incoming ions. Instrumental variables were controlled by an Extrel FTMS Odyssey data station. Switching between normal excitation and detection and quadrupolar excitation, where $\mathrm{rf}$ is applied to all four excite-receive plates of the ICR cell, was achieved by a relay "switch box" similar to that described by other workers [19]. The circuitry employed to produce simultaneous dipolar SORI and broadband quadrupole excitation is described in detail elsewhere [13]. Broadband quadrupole excitation was accomplished with repeated chirp waveforms that covered the selected frequency range. The broadband quadrupole excitation waveforms were generated by software developed at our laboratory and produced with a LeCroy (Chesnut Ridge, NY) arbitrary function generator. For SORI experiments, single-frequency quadrupole cooling was performed at $\mathrm{m} / \mathrm{z} 3790$ during ion injection for massto-charge ratio selective-ion accumulation of the $14+$ charge state of the streptavidin tetramer. This process was followed by application of a dipolar rf excitation pulse $+1000 \mathrm{~Hz}$ off-resonance relative to the $14+$ charge state for $2 \mathrm{~s}$ with broadband quadrupole excitation over a large mass-to-charge' ratio range (10006500). Nitrogen gas was introduced into the cell region as a target gas during both the ion accumulation and subsequent quadrupole cooling SORI events. For SORI experiments, the amplitude of the excitation pulse was varied throughout a $110-360-\mathrm{mV}_{\mathrm{p}-\mathrm{p}}$ range to provide sufficient fragmentation of the tetramer. Singlefrequency and broadband quadrupole excitation amplitudes were generally $1-2 \mathrm{~V}_{\mathrm{p}-\mathrm{p}}$. Data acquisition times for each spectrum were typically $0.5 \mathrm{~s}$.

\section{Results and Discussion}

The results for heating-activation in the ESI atmosphere-vacuum interface region for the intact noncovalent multimeric proteins avidin, concanavalin $A$, and adult human hemoglobin were reported previously [3]. Utilization of relatively gentle interface conditions allowed the intact complexes to be detected under conditions that also provided sufficient desolvation of the large protein ions. Adjustment of instrumental variables enabled the interface conditions to be made harsher and thermal and/or collisional energy could be increased. Specifically, increased heat applied to the metal inlet capillary to cause TID of the tetramers produced a series of charge states for the monomer and trimer species. In the case of hemoglobin, harsher interface conditions produced by higher capillaryskimmer offset voltages also resulted in formation of trimers because of the relatively labile tetrameric association of this protein compared to avidin and Con $\mathrm{A}$. These relatively low charge state high mass-to-charge ratio trimers appeared to correlate with the appearance of monomers at much lower mass-to-charge ratio. A mechanism was proposed to account for these observations in which two different pathways for monomer ion formation were possible [3]. The highest charge state monomer ions were attributed to the earlier breakup of the tetramer before the charge state was "locked in," that is, before desolvation was complete $[1 i, 3]$. It was suggested also that the monomer might lose portions of its higher order structure, be "elon- 
gated" through a coulombically driven mechanism, and then be ejected from the aggregate early in the ion desolvation process. This behavior would allow the monomer to exhibit a higher charge-to-mass ratio due to its relaxed structure and greater availability of charge sites. In addition, gas-phase dissociation of the tetramer (after initial ion formation-charge state determination) could account for the lower charge state monomer ions and trimer species observed [3], which have approximately complementary charge state distributions. Other tentative evidence for formation of the trimer species late in the ion transport process comes from their stability to additional activation in the interface region once formed, that is, increased $\Delta \mathrm{CS}$ or capillary heating does not dissociate the trimers.

\section{Electrospray Ionization Interface Effects on Streptavidin Dissociation}

The positive ion ESI mass spectrum of streptavidin in a $\mathrm{pH}$ 8.6 10-mM ammonium acetate solution under gentle ESI interface conditions (capillary heat of $20 \mathrm{~W}$, $\sim 210{ }^{\circ} \mathrm{C}$, and a $\Delta \mathrm{CS}$ of $70 \mathrm{~V}$ ) yields a prominent tetramer ion distribution $\left(\mathrm{Q}^{16+}\right.$ to $\left.\mathrm{Q}^{1++}\right)$ as shown in Figure 1. The $M_{\mathrm{r}}$ determination of 53,140 $\pm 60 \mathrm{u}$ from the experimental data for the three charge states is in reasonable agreement with the expected value of 53,084 $\mathrm{u}$ calculated from the monomer weight $\left(M_{\mathrm{r}}=13,271\right.$ u) for this particular sample, with consideration of the low instrumental resolution and likely contributions due to solvation and/or adduction. The spectrum is consistent with the specific subunit association expected from solution behavior and exhibits no random aggregation of monomer. This result supports our earlier studies and shows that these specific associations can be observed under proper interface conditions. The relatively clean spectrum and high signal-to-noise ratio can be attributed to better purification of this protein compared to our previous studies. In our experience, commercial preparations of streptavidin often display

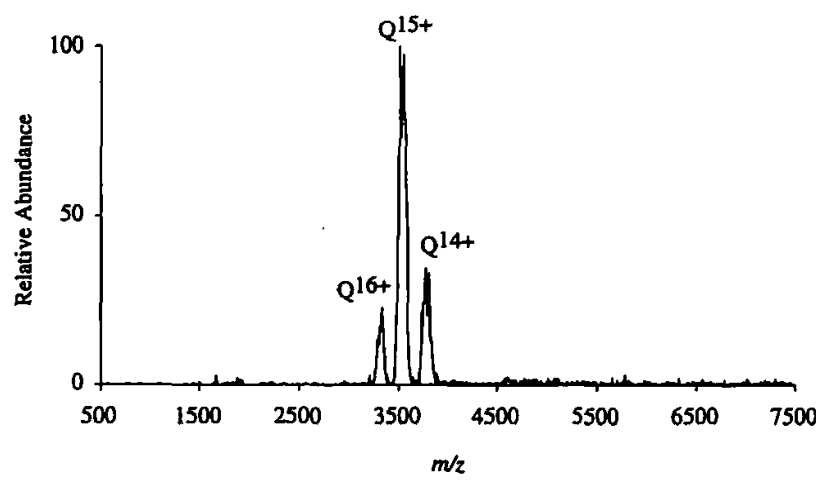

Figure 1. Positive ion ESI mass spectrum obtained on an extended mass-to-charge ratio range quadrupole for streptavidin in $\mathrm{pH} 8.610-\mathrm{mM}$ ammonium acetate under gentle interface conditions, that is, capillary heating of $20 \mathrm{~W}\left(\sim 210^{\circ} \mathrm{C}\right)$ and $\Delta \mathrm{CS}=70$ V. much weaker ion signals in addition to unresolvable peaks for the tetramer in the ESI spectra.

Harsher interface conditions produced by increasing the heating applied to the metal inlet capillary to $26 \mathrm{~W}\left(\sim 253^{\circ} \mathrm{C}\right)$ result in thermally induced dissociation of the streptavidin tetramer into monomer and trimer species, as previously reported for the tetrameric proteins avidin, $\mathrm{Con} \mathrm{A}$, and $\mathrm{Hb} \mathrm{A}_{\mathrm{o}}$ (Figure 2). Ions indicative of the tetramer are still apparent, but the monomer peaks (labeled $\mathrm{M}^{9+}$ to $\mathrm{M}^{5+}$ ) centered at $m / z 1900$ are the most abundant for the hotter capillary conditions. Also evident are peaks at higher massto-charge ratio $(\mathrm{m} / \mathrm{z} 4400-5600)$ that were previously attributed to trimers (labeled $\mathrm{T}^{9+}$ to $\mathrm{T}^{7+}$ ). The center of the product ion distributions for the monomer species at $7+$ and trimer at $8+$ are consistent with the breakup of the $15+$ tetramer charge state. Tandem mass spectrometry experiments were then performed by FTICR to investigate the gas-phase decomposition of the tetramer.

\section{Fourier Transform Ion Cyclotron Resonance Experiments with Streptavidin}

The FTICR mass spectrum for streptavidin indicates prominent charge states $\left(\mathrm{Q}^{15+}\right.$ to $\left.\mathrm{Q}^{13+}\right)$ for the specific subunit association as a tetramer (Figure 3 ) with an experimental $M_{\mathrm{r}}$ determination of $53,084 \pm 5 \mathrm{u}$ for the three charge states, in exact agreement with that expected. There appears to be little or no solvation/adduction to the tetrameric charge states, which is different from the quadrupole data and therefore may be a result of the trapping and/or detection process. Interestingly, the observed charge state distribution is narrow, as observed in the quadrupole studies, but shifted lower by approximately one charge. To compare TID studies that employed a heated metal capillary by using the extended mass-to-charge ratio quadrupole instrument with tandem mass spectrometry data acquired by FTICR, a SORI experiment was conducted to induce dissociation of a selected tetrameric charge state. Selected-ion accumulation [13]

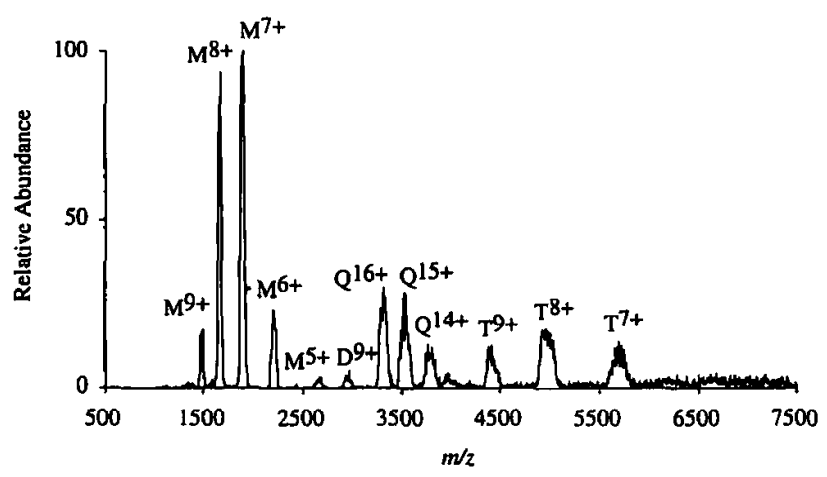

Figure 2. ESI mass spectrum for thermally induced dissociation of streptavidin obtained on an extended mass-to-charge ratio range quadrupole under harsh interface conditions, that is, capillary heating of $26 \mathrm{~W}\left(\sim 253^{\circ} \mathrm{C}\right)$. 


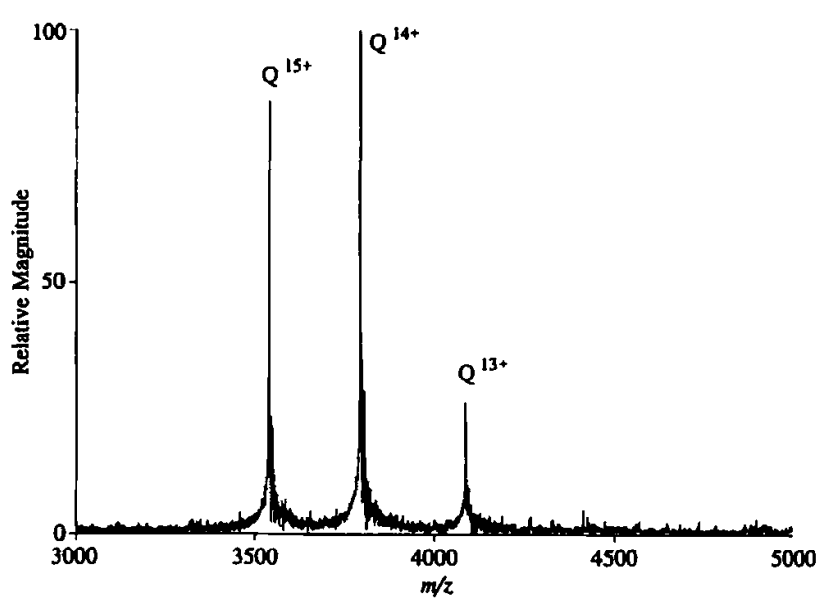

Figure 3. ESI FTICR mass spectrum of streptavidin in $\mathrm{pH} 8.6$ 10-mM ammonium acetate.

was performed on the $14+$ charge state by implementation of single-frequency quadrupole excitation during the ion injection and pulsed gas inlet periods. The utility of selected-ion accumulation for enhancement of precursor ion abundance is well demonstrated by these studies, with an observed increase in signal-to-noise ratio of approximately a thousandfold for the $14+$ charge state. However, it must be noted that this factor of enhancement is arbitrary and can be increased until the space-charge limit is reached-a function of all charged species in the cell. SORI was conducted on the tetrameric charge state by applying a $265-\mathrm{mV}$ dipolar If excitation pulse at $1000 \mathrm{~Hz}$ above the cyclotron frequency of the selected charge state, combined with broadband quadrupole excitation over a $m / z$ 1000-6500 range. The resulting mass spectrum (Figure 4) indicates decomposition of the $14+$ tetramer charge state species into either $7+(m / z 1897)$ or $6+(m / z$ 2213) monomers and $7+(m / z 5692)$ or $8+(m / z$

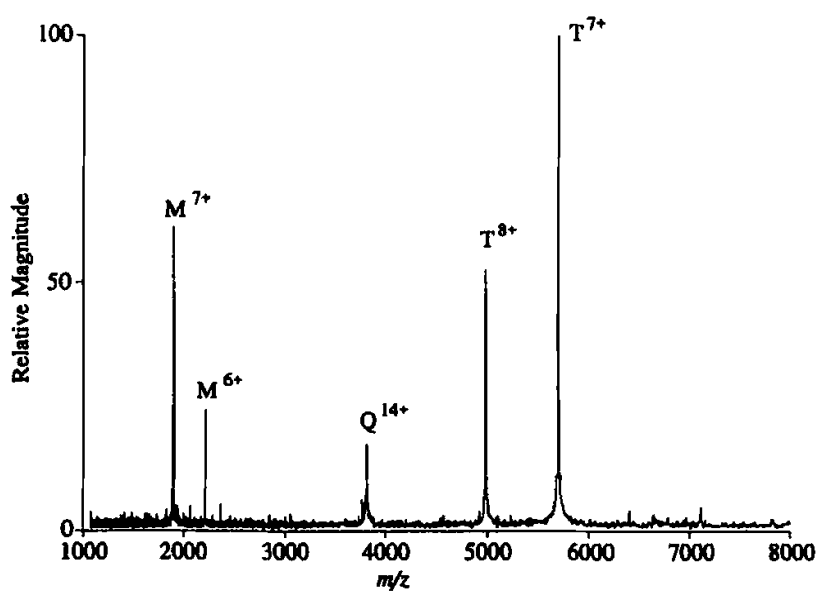

Figure 4. SORI FTICR mass spectrum for the $14+$ charge state of the streptavidin tetramer. This spectrum represents the massto-charge ratio selected ion accumulation of the $14+$ tetrameric charge state with subsequent $265-\mathrm{mV}$ SORI at $+1000 \mathrm{~Hz}$ and broadband quadrupole excitation from $\mathrm{m} / \mathrm{z} 1000$ to 6500 .

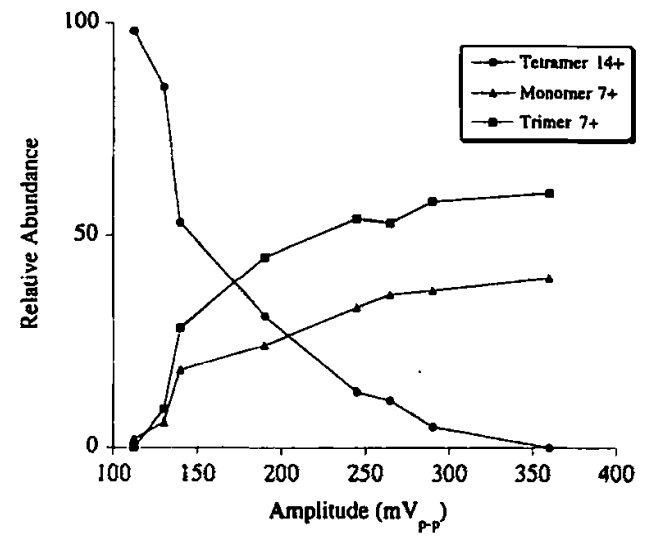

Figure 5. Effect of SORI pulse amplitude (millivolts peak to peak) on dissociation of the selected $14+$ tetrameric charge state of streptavidin.

4979) trimer species, consistent with the in-source TID results on the protein. These tandem mass spectrometry results provide proof for the-hypothesis that the trimers result from gas-phase decomposition of the tetramer and are complementary to monomer ion formation. Furthermore, an energy dependence study was performed by alteration of the rf amplitude of the dipolar excitation event to examine the effect on the gas-phase dissociation of the tetramer. Consistent with the expected behavior, as more energy is deposited into the tetramer, its abundance decreases simultaneously with an increase in production of monomer and trimer species (Figure 5). SORI excitation of the $14+$ charge state at $112 \mathrm{mV}$ produced no significant evidence of product ions. As the excitation pulse amplitude was increased, the tetramer species was observed to dissociate into the complementary monomer and trimer ions, with complete dissociation into products at an amplitude of $360 \mathrm{mV}$. Varying the duration of the SORI pulse from 2 to $20 \mathrm{~s}$ at a constant amplitude of $140 \mathrm{mV}$ had no noticeable effect on the extent of fragmentation.

\section{Gas-Phase Tetrameric Dissociations}

Earlier studies with other tetrameric noncovalent proteins $[1 \mathrm{~m}, 3]$, and the recent TID studies and SORI FTICR experiments on streptavidin, show unambiguously that the trimers are a result of gas-phase decomposition of the tetramer. The monomer ions of complementary charge state are also observed, which indicates that the tetramer (4M) dissociates into monomer $(\mathrm{M})$ and trimer $(3 \mathrm{M})$ components:

$$
[4 \mathrm{M}+14]^{14+} \rightarrow[\mathrm{M}+7 \mathrm{H}]^{7+}+[3 \mathrm{M}+7 \mathrm{H}]^{7+}
$$

in strong preference to the possible pathway that produces the dimer $(2 \mathrm{M})$ species:

$$
[4 \mathrm{M}+14]^{14+} \rightarrow 2[2 \mathrm{M}+7 \mathrm{H}]^{7+}
$$


There are several physical models that may qualitatively explain the preferential asymmetric breakup of a tetrameric complex into unequal portions (i.e., 1:3) rather than into identical portions (i.e., 2:2). The first model draws an analogy to the breakup of a charged liquid droplet, such as produced by electrospray. Ryce and Wyman [20] discussed the breakup of charged droplets in terms of a "fissionability" parameter $x$. A fissionability of $x=1$ corresponds to the Rayleigh instability condition for a charged droplet. When $x=1$ the droplet will spontaneously break up into smaller droplets without an energy barrier. However, for $x<1$ it is sometimes possible for a droplet to be unstable, albeit with an energy barrier separating the initial and final states. In this case, division into unequal droplet volumes is energetically preferred. Although a protein complex is not fully equivalent to a charged droplet, there is a qualitative similarity because in both cases the system is held together by short-range forces, such as hydrogen bonding, and destabilized by the longrange forces of coulombic repulsion [21]. Therefore, the physical behavior of the two systems should have qualitative similarities, such as their asymmetric breakup. The charged droplet analogy also may give some insight as to why the relative charge of the monomer is much higher than that of the trimer. In the dissociation of the present protein, the products have a $1: 3$ mass ratio. If we assume that mass is proportional to volume and model the products as charged spheres, the ratio of their radii is given by $\lambda=r_{1} / r_{2}=$ $\left(m_{1} / m_{2}\right)^{1 / 3}=(1 / 3)^{1 / 3}=0.693$. In their model, Ryce and Wyman [20] defined a parameter $\alpha$ as the relative separation between the centers of the spheres at a "psuedo saddle point" of the energy surface for separating spheres. In effect, $\alpha$ is an adjustable parameter that describes the point at which charge redistribution between the two spheres can no longer occur. For example, if this occurs when the spheres are just touching, then $\alpha=1$. For larger separations, $\alpha$ increases proportionally. Ryce and Wyman [20] also used a parameter $p$, defined as $[a \lambda+\alpha-1] /[a+(\alpha-1) \lambda]$, and according to their equation $q_{1} / q_{2}=\lambda p$. From these equations, one calculates that for division into a monomer and a trimer, the ratio of $\left(m_{1} / z_{1}\right) /\left(m_{2} / z_{2}\right)$ varies between 0.48 for $\alpha=\infty$ and 0.69 for $\alpha=1$. These numbers are all less than 1 , which implies that in the liquid drop model, the smaller fragment (i.e., monomer) should get more than its share of the charge, which is the same trend that was observed experimentally. The experimental observations shown in Figure 4 place the $\left(m_{1} / z_{1}\right) /\left(m_{2} / z_{2}\right)$ ratio closer to 0.4 , so this simple theory somewhat underestimates the magnitude of this effect. If the structure of the expelled monomer is significantly perturbed, for example, "extended," as speculated previously [3], the relatively greater amount of monomer charge also could be explained. Other more subtle factors also may contribute to the present observations, such as entropic effects. For instance, a difference in entropy for fragmentation into products with a 1:3 mass ratio, rather than a 1:1 mass ratio, is small $(\sim 0.29 R)$, but also may contribute to the observed behavior.

\section{Conclusions}

The consistency in results obtained for streptavidin by TID studies on the extended mass-to-charge ratio range quadrupole instrument and SORI dissociation studies with FTICR provide unambiguous evidence for the asymmetric decomposition of electrosprayed multimeric ions. It appears that the dissociation of multiply charged tetrameric proteins produced by ESI, activated by either collisional or thermal means into the gas phase, generally results in dissociation into unequal portions and represents the lowest energy pathway. The removal of one subunit, that is, monomer, from the tetramer results in a remaining trimer portion with substantially lower average charge per subunit than the tetramer, as well as the more highly charged monomer, and is the preferred mechanism of decomposition for a multimer composed of four identical subunits. These results provide insight into the dissociations of noncovalent tetrameric ions formed by electrospray and possibly into the electrospray process itself. They also stress the exciting application of FTICR for the study of large noncovalent proteins and other complexes and to probe their dissociations, which perhaps eventually may lead to the use of FTICR to provide more extensive information with regard to the structure of large gas-phase biomolecular ions.

\section{Acknowledgments}

We thank the Laboratory Directed Research and Development of Pacific Northwest Laboratory for support of this research through the U.S. Department of Energy. Pacific Northwest Laboratory is operated by Battelle Memorial Institute for the U.S. Department of Energy through contract DE-AC06-76RLO 1830. We also thank Dr. Karen J. Light-Wahl for encouragement of these studies and Dr. David C. Gale for helpful suggestions.

\section{References}

1. (a) Ganem, B.; Li, Y.-T.; Henion, J. J. Am. Chem. Soc. 1991, 113, 6294; (b) Ganem, B.; Li, Y.-T.; Henion, J. J. Am. Chem. Soc. 1991, 113, 7818; (c) Katta, V.; Chait, B. T. J. Am. Chem. Soc. 1991, 113, 8534; (d) Baca, M.; Kent, S. B. H. J. Am. Chem. Soc. 1992, 114, 3992; (e) Ganguly, A. K.; Pramanik, B. N.; Tsarbopoulos, A.; Covey, T. R.; Huang, E.; Fuhrman, S. A. J. Am. Chem. Soc. 1992, 114, 6559; (f) Smith, R. D.; Light-Wahl, K. J.; Winger, B: E.; Loo, J. A. Org. Mass Spectrom. 1992, 27, 811; (g) Jaquinod, M.; Leize, E.; Potier, N.; Albrecht, A.-M.; Shanzer, A.; Van Dorsselaer, A. Tetrahedron Lett. 1993, 34, 2771; (h) Ganem, B.; Henion, J. D. Chem. Tracts-Org. Chem. 1993, 6, 1; (i) Smith, R. D.; Light-Wahl, K. J. Biol. Mass Spectrom. 1993, 22, 493; (j) Huang, E. C.; Pramanik, B. N.; Tsarbopoulos, A.; Reichert, P.; Ganguly, A. K.; Trotta, P. P.; Nagabhushan, T. L.; Covey, T. R. J. Am. Soc. Mass Spectrom. 1993, 4, 624; (k) Li, Y.-T.; Hsieh, Y.-L.; Henion, J. D.; Ganem, B. I. Am. Soc. Mass Spectrom. 1993, 4, 631; (1) Loo, J. A.; 
Holsworth, D. D.; Root-Bernstein, R. S. Biol. Mass Spectrom. 1994, 23, 6; (m) Tang, X.; Brewer, C. F.; Saha, S.; Chernushevich, I.; Ens, W.; Standing, K. G. Rapid Commun. Mass Spectrom. 1994, 8, 750 .

2. Light-Wahl, K. J;; Winger, B. E.; Smith, R. D. I. Am. Chem. Soc. 1993, 115, 5869.

3. Light-Wahl, K. J.; Schwartz, B. L.; Smith, R. D. J. Am. Chem. Soc. 1994, 116, 5271.

4. (a) Loo, J. A.; Udseth, H. R.; Smith, R. D. Rapid Commun. Mass Spectrom. 1988, 2, 207; (b) Smith, R. D.; Loo, J. A.; Barinaga, C. J.; Edmonds, C. G.; Udseth, H. R. J. Am. Soc. Mass Spectrom. 1990, 1, 53; (c) Rockwood, A. L.; Busman, M.; Udseth, H. R.; Smith, R. D. Rapid Commun. Mass Spectrom. 1991, 5, 582.

5. Argaraña, C. E.; Kuntz, I. D.; Birken, S.; Axel, R.; Cantor, C. R. Nucleic Acids Res. 1986, 14, 1871.

6. Wilchek, M.; Bayer, E. A. Anal. Biochem. 1988, 171, 1.

7. (a) Schwartz, B. L.; Light-Wahl, K. J.; Smith, R. D. J. Am. Soc. Mass Spectrom. 1994, 5, 201; (b) Schwartz, B. L.; Light-Wahl, K. J.; Smith, R. D. Proceedings of the 42nd ASMS Conference on Mass Spectrometry and Allied Topics; Chicago, IL, May 29-June 3, 1994; $\mathrm{p} 19$.

8. (a) Henry, K, D.; Quinn, J. P.; McLafferty, F. W. I. Am. Chem. Soc. 1991, 113, 5447; (b) Beu, S. C.; Senko, M. W.; Quinn, J. P.; McLafferty, F. W. I. Am. Soc. Mass Spectrom. 1993, 4, 190.

9. Bruce, J. E.; Anderson, G. A.; Hofstadler, S. A.; Van Orden, S.
L.; Sherman, M. S.; Rockwood, A. L.; Smith, R. D. Rapid Commun. Mass Spectrom. 1993, 7, 914.

10. Gauthier, J. W.; Trautman, T. R.; Jacobson, D. B. Anal. Chim. Acta 1991, 246, 211.

11. Guan, S.; Wahl, M. C.; Marshall, A. G. J. Chem. Phys. 1994, $100,6137$.

12. Huang, Y.; Pasa-Tolic, L.; Guan, S.; Marshall, A. G. Anal. Chem. 1994, 66, 4385.

13. Bruce, J. E.; Van Orden, S. L.; Anderson, G. A.; Hofstadler, S. A.; Sherman, M. S.; Rockwood, A. L.; Smith, R. D. J. Mass Spectrom. 1995, 30, 124.

14. Chilkoti, A.; Tan, P. H.; Stayton, P. S. Proc. Natl. Acad. Sci. USA 1995, 92, 1754.

15. Winger, B. E.; Light-Wahl, K. J.; Ogorzalek Loo, R. R.; Udseth, H. R.; Smith, R. D. J. Am. Soc. Mass Spectrom. 1993, 4, 536.

16. Chowdhury, S. K.; Katta, V.; Chait, B. T. Rapid Commun. Mass Spectrom. 1990, 4, 81.

17. Gale, D. C.; Smith, R. D. Rapid Commun. Mass Spectróm. 1993, 7, 1017.

18. Winger, B. E.; Hofstadler, S. A.; Bruce, J. E.; Udseth, H. R.; Smith, R. D. J. Am. Soc. Mass Spectrom. 1993, 4, 566.

19. Speir, J. P.; Gorman, G. S.; Pitsenberger, C. C.; Turner, C. A.; Wang. P. P.; Amster, I. J. Anal. Chem. 1993, 53, 1746.

20. Ryce, S. A.; Wyman, R. R. Can. J. Phys. 1970, 48, 2571.

21. Rockwood, A. L.; Busman, M.; Smith, R. D. Int. I. Mass Spectrom. Ion Processes 1991, 111, 103. 